\title{
Identification of intelligent substation multi-source data based on extended SCD panoramic model
}

\author{
HOU Aijun ${ }^{1, a}$, LIU Junhong ${ }^{2, b}$, HU Chunchao ${ }^{1, c}$, MA Kai $^{1, d}$ \\ ${ }^{1}$ Power Science Research Institute of Guangdong Power Grid Co., Guangzhou 510080, China \\ ${ }^{2}$ Beijing Sifang Automation Co. Ltd. ,Beijing 100085, China \\ ahouaijun@139.com, ${ }^{\mathrm{b}}$ liujunhong@sf-auto.com, chuchunchao@139.com, ${ }^{\mathrm{d}}$ makai@139.com
}

Keywords: intelligent substation; data identify; PMU model; SSD applications

\begin{abstract}
Analyzing and dealing with massive data in time to provide proper and reliable data for advanced applications of substation is one of the main tasks of intelligent substation. This paper introduced the quality identify of multi-source data in detail based on the panoramic SCD model which intergrated the SSD model. According to this SSD model, Remote measure and communication and data identify have been realized through extracting topology and substation's primary equipment's data. Three phase voltage and current have been identified, balance level of single point such as transformer, bus ,switch and three phasor unbalanced degree have been judged by multi-sources in this thesis. The data identify method is valid and superior due to the correlation to the secondary equipment and the special PMU data transmission mode.
\end{abstract}

\section{Introduction}

With the rapidly increase of the measuring data and advanced applications, the data needs to deal with in substation increase greatly too and the vast data can not upload fully to dispatch center. So it is becoming more and more important to identify the vast data.. Identification and analysis of substation's panoramic data is urgent now. It will provide accurate and reliable data for advanced application of intelligent substation by detecting, identifying, checking and analasys data.

The data identification in the thesis is based on the panoramic SCD model which not only includes primary device data, but also includes secondary equipment data. The integration of measurement and control PMU node uploads dynamic data through a dedicated channel with 1344 protocol, at the same time, it also uploads effective value by MMS message through intelligent substation's bay MMS net. This paper introduces data identification by multi-source data in SCADA, judgment of single point balance of station transformer, busbar, switch and so on and three phasor balanced degree judgment. If the single point judgment is abnormal, multipoint and comprehensive judgment again deep data identification is introduced also.

\section{The substation panoramic SCD model based on extended SSD model}

\section{Introduction to panoramic SCD model}

The model of Substation bay's information which is used for measurement, control and protection is built according to the IEC61850 standard. System configurator incorporates ICD files from substation bay equipment such as protect, measurement and control, PMU and other public auxiliary control IED to generate system configuration file for the whole substation. Through analyzing the complete SCD model, information sheet about the whole primary and secondary equipment can be built in the database. The information include electric parameters, location, DC value, state monitoring value, measurement value, etc., also include the basic data of protection and communication system such as protection parameters, events, and ranging, setting value and the parameters of the wave record file, etc. By Importing data from the SCD file into the database, the system configurator can output the entire second SCD model of intelligent substation equipment.

In all the domestic existing system configurator, information input and output of the SSD model are not supported. At the same time, in the IEC61850 standard, according to the description of the 
SSD, it only defines the association between primary device and secondary device logical nodes (lnClass). Tthe specific gauge point information is not defined. To build a panoramic view of SCD model with the full SSD information, the current IEC61850 standard needs to be expanded to set a set of SSD model specifications and to generate a complete substation SCD model information ${ }^{[1]}$

\section{Introduction of SSD model related to secondary equipment}

In the SSD model information, IEC61850 standards only defines the logical nodes associated with the secondary equipment level (lnClass = "MMXU") for primary equipment information definition, but not defines the specific gauge point information. this association is different from the IEC61970 CIM model definition which has instance gauge point configration in the CIM model. So it is needed to define all the gauge point of the secondary equipment logic nodes mapping relation in the primary equipment logic nodes in SSD to search all the measurement information corresponding to primary equipment . But the SCD has existed all logical nodes and data object's definition, measuring point defined in SSD needs to adopt the way of reference to index data object of secondary equipment ${ }^{[4]}$.

Based on SSD Model, primary equipment topology for transformer substation is analyzed, and the analysis results are stored in real-time database. Topology analysis module adopts the algorithm of local trends to realize the local flow calculation of transformer substation. trend for each key device Node is analyzed by the secondary equipment measurement real value corresponding to primary equipment in SSD. Active and reactive power, etc. of the branch are calculated to provide follow-up data identification and other advanced application to realize data identification of measurement value of each branch and component, Intelligent alarm etc.

\section{Multi professional data fusion by SSD model}

Sample model as follow, iedName $=$ "CL2201" is measuring and control device, iedName="PL2201A"is A protection device, iedName="CL220B"is B protection device, iedName=“CSD326PMUXKL”is PMU device, each represent automation specialty, protection specialty, PMU data.

Sample model:

$<$ Bay name="220kV2211XL">

$<$ ConductingEquipment name="2211" type="CBR">

$<$ LNode iedName="CL2201" prefix="' lnClass="CSWI" lnInst="0"/>

$<$ LNode iedName="CL2201" prefix="" lnClass="MMXU" lnInst="11" doi="A.phsA"/>

$<$ LNode iedName="PL2201A" prefix="" lnClass="MMXU" lnInst="1" doi="A.phsA"/>

$<$ LNode iedName="PL2201B" prefix="" lnClass="MMXU" lnInst="1" doi="A.phsA"/>

$<$ LNode iedName="CSD362PMUXKL" prefix="" lnClass="MMXU" lnInst="1" doi="A.phsA"/>

$<$ LNode iedName="CL2201" prefix="" lnClass="MMXU" lnInst="11" doi="PhV.phsA"/>

$<$ LNode iedName="PL2201A" prefix="' lnClass="MMXU" lnInst="2" doi="PhV.phsA"/>

$<$ LNode iedName="PL2201B" prefix="" lnClass="MMXU" lnInst="1" doi="PhV.phsA"/>

$<$ LNode iedName="CSD362PMUXKL" prefix="' lnClass="MMXU" lnInst="1" doi="PhV.phsA"/>

$<$ LNode iedName="CL2201" prefix="Go1CB" lnClass="CSWI" lnInst="1" doi="Pos"/>

For data from different major, same doi represents the same electric data comes from the same equipment. For example, “A.phsA”represents current of phase A, "PPV.phsA” represents voltage of phase A.

thus, four different resources data comes from three majors fused by SSD model. So does other primary equipment's electric value, status value, alarm information.

\section{Primary equipment topological connection by the SSD model}

SSD model through primary device's Terminal 's attribute "connectivityNode" can realize the SSD model topology. In SSD model, primary equipment such as transformer, breaker, switch all have terminal nodes. The terminals which have the same attribute value of connectivityNode represents they are connected by wire. Start form the entrance equipment, look up the same connectivityNode value terminal, all Connection with the terminal will be connected until the bus 
and transformer equipment, thus formed topological connection from entrance wire to the transformer. Again from high voltage, medium voltage, low voltage winding's terminal, the whole topology of the substation can be formed according to this method .

\section{Introduction to PMU using the MMS channel to upload}

PMU devices in the substation, the same as secondary protection IED devices, as an intelligent IED node, the PMU ICD model instance into logical LD in SCD. Different from the conventional dynamic data sent by PMU, integrated monitoring and control PMU node, in addition to the dedicated channel with a 1344 protocol to send dynamic data, but also through the spacer layer MMS network of intelligent station upload the RMS by MMS messages . The RMS is calculated by PMU through a certain algorithm for dynamic data of each acquisition channel and is provided directly to the substation monitoring system and far motivations for using by monitoring network. Generally, the transmission frequency is per one second or a few second. RMS data includes: three-phase current (SeqA), voltage (SeqV) amplitude (cVal.mag), angle (cVal.ang), data quality (q), active (TotW), reactive power (TotVAr), frequency, more than the data transferring by routine PMU.

On the intelligent monitoring substation net, the information general surveillance systems, remote devices can access are usually MMS message information sent by secondary device on the spacer layer. So RMS data model of PMU sent by the MMS is used.

\section{Data Identification}

\section{The overall data flow of Identification}

First, identify each telemetry data of multiple sources, if the data is normal, no further analysis is needed; if identification abnormal, identification for three phase voltage and current data of single point monitoring and control data is needed so that you can analyze the reliability of the data. If the voltage and current data are normal, we can conduct a comprehensive analysis to identify the device P, Q, COS, switch position and other data.

\section{Quality identification of multi-source data}

According to SSD files, extract topology and primary device data information and on this basis to achieve telemetry, remote signaling and other data quality identification.

First through different sources of measurement data, identificate the measurement data of SCADA, judgments based:

Ua $($ scada source $)=$ Ua $($ prot source $)=$ Ua $($ PMU source $)$

Ia $($ scada source $)=$ Ia (prot source) $=$ Ia (PMU source)

Example model as described in Section 1.2.1 below. As show in the example model, the A-phase voltage measurement data is checked for the switch "2211". Switch associated with the following four IED equipment: Gage CL2201, protection A PL2201A, protection B PL2201B, PMU device CSD362PMUXKL; So there are four voltage measuring sources and three categories of data source: monitoring and control, protection, PMU. When checking data, measuring point "PhV.phsA" in the four IED "MMXU" function node can be found from the model (this model instances, respectively, under the four IED associated equipment, phase A voltage associated are identical). In the real-time acquisition systems, when real-time data updates in the database, the data identification module can directly navigate to the corresponding node voltage data in real time according to the correlation in thel model.

By reading protection data "PhV.phsA" and PMU's voltage measurement for further checking of SCADA voltage on the switch 


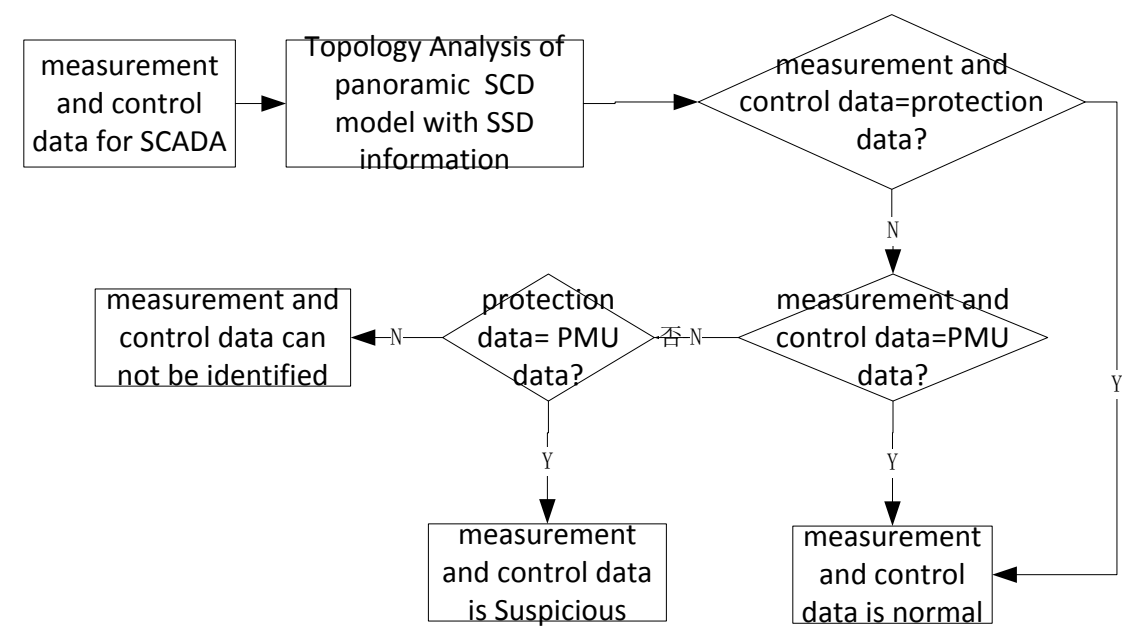

Fig.1 Quality identification of multi-source data

\section{Single point balance judge for each measurement points}

Detection methods: balance tests mainly on the wire and circuit, bus, each side of the transformer as following:

$\mathrm{Uab}=\mathrm{Ubc}=\mathrm{Uca}$

$\mathrm{Ua}=\mathrm{Ub}=\mathrm{Uc}$

$\mathrm{Ia}=\mathrm{Ib}=\mathrm{Ic}$

If the three-phase voltage / current difference is less than the threshold value (calculated by $20 \%$ ) ,three phase is even, otherwise uneven.

A, B, C three-phase voltage balance detection as example, the main process is as follows:

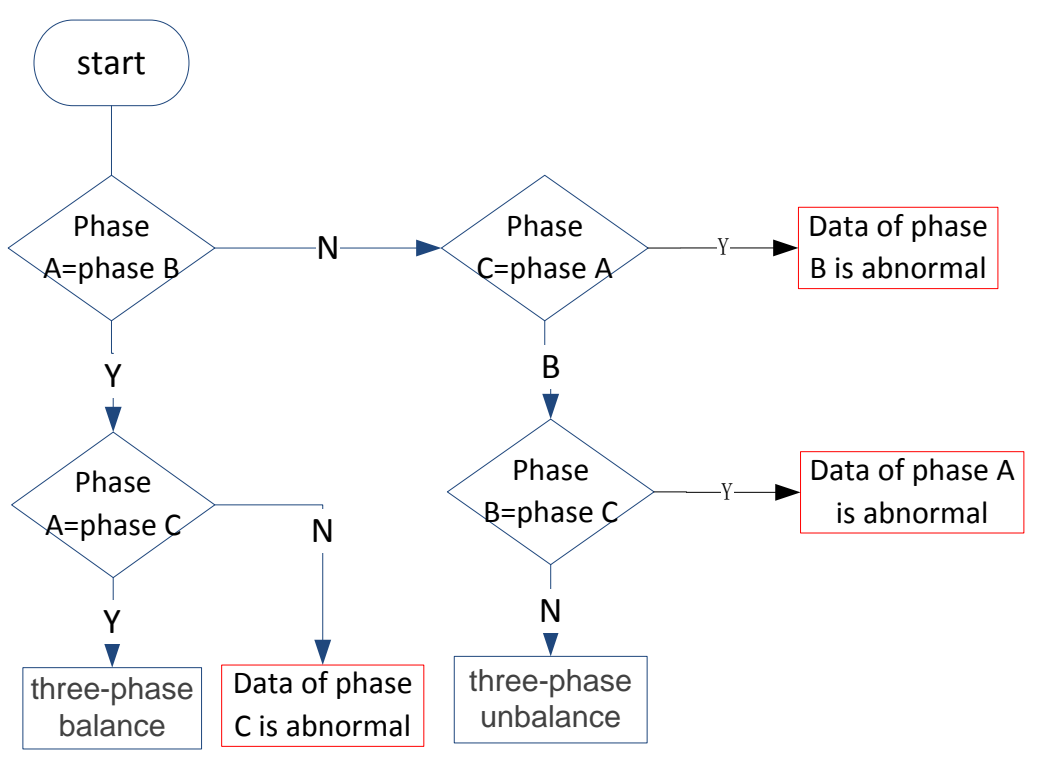

Fig2. Single point balance judge for each measurement points

Because data identification application modules used multi-source data, the abnormal data can be correctly alarmed after checking in the intelligent alarm module. Alarms may occur: Unbalanced: A-phase or three-phase unbalanced: B-phase or three-phase unbalance: C-phase or three phase imbalance: ABC phase.

After the identification of the single point of data, you can enter a subsequent multi-point and comprehensive analysis on the basis of the recognition result.

\section{Summary}

Currently primary equipment of power system will gradually implement intelligent, advanced applications increased and the substation monitoring data are gradually rising. Data identification 
function requires continuous development and improvement. This paper describes the quality identify of multi-source data in detail based on the panoramic SCD model which intergrated the SSD model. By measuring the different sources of data, the SCADA measurement data identification is realized, including three-phase phase voltage, phase current, line voltage, line current checking, as well as single point balance and three-phase balance judgment on measurement data of the transformer, bus, switches, etc.. Finally, the upload strategy of recognition result is introduced to ensure the data send to dispatch accurate and comprehensive.

\section{Reference}

[1] Haiyang Yu. Advanced applications exploration based on smart substation multivariate data [J] Northeast Electric Power Technology, 2014, (6): 38-40.

[2] Wanjian Bai. Data identification based on intelligent substation integration monitoring system [J] Shandong Power Technology, 2014,41 (6): 1-4.

[3] Mingzhu Chen. Advanced application Status and Prospects of Intelligent Substation [J] Hubei Power, 2011,35: 86-89.

[4] Bingjie Wang. Data identify applications and development on intelligent substation integrated monitoring system [D] Jinan: Shandong University, 2014.

[5] Q / GDW 3832009 Technical Guidelines for intelligent substation [S]. Beijing: State Grid Corporation, 2009.

[6] Ting Lei, Chuanbo,Zhu, Taigui Huang, etc Research on data quality identification method based on automation platform [J]. power system automation, 2012,36 (12):.. 71-75.

[7] Ying Li, Renmu He, Yan Xu Guangdong power grid data identification research on PMU load model [J] China Southern Power Grid Technology, 2009,3 (1): 16-19. 